\title{
OP-03 MATERNAL HEALTH SERVICES IN THE TRIBAL COMMUNITY OF BALASORE DISTRICT, ODISHA: CHALLENGES AND IMPLICATIONS
}

Ranjit Kumar Dehury. Goa Institute of Management, Panaji (Goa), India

10.1136/bmjgh-2016-EPHPabstracts.3

Background Odisha is a 'high-focus' state, a category set up in the National Rural Health Mission, as it has high maternal mortality ratio. The state is still far away to achieve the Millennium Development Goal of reducing maternal mortality ratio to 109 by 2015 . While tribal population constitutes $22.9 \%$ of Odisha's total population, maternal mortality among tribal women is observed to be significantly high. Failure to adequately promote maternal health services including Janani Surakbya Yojna (JSY) has accentuated poor maternal health condition in tribal pockets. Since maternal health is largely dependent on the health infrastructure, consorted effort of the service provider, creating scope to integrate indigenous practice with modern medicine and evidence-based decision-making using Health Management Information System is critical. The broad objective of this study is to appraise maternal health services of tribal areas that are remotely situated by using medical as well as non-medical parameters. Specifically, the study makes an assessment of the infrastructural capacity and competence of the frontline health worker, Accredited Social Health Activist (ASHA), for promotion of maternal health. Our study also captured the perceptions and expectations of the local tribal community during childbirth. Further, the study evaluated the Health Management Information System for integral planning and monitoring of maternal health in tribal community for transformation of JSY programme.

Methods We studied Jaleswar block of Balasore district, which is declared as a Modified Area Development Approach block by the government of India due to its high concentration of tribal population and geographical inaccessibility because of its riverine feature. In order to assess the infrastructural capacity related to maternal health, the study used 'Parijata tool' developed by the United Nations Children's Fund but with modification for regional suitability. The assessment tool covered number of parameters like manpower strength, availability of drugs and consumables and procedure for clinical practices. Additionally a checklist was developed for assessing competency of ASHA and their involvement in promoting maternal health as per national guidelines. Our study has specifically looked into how ASHAs promoted health services in congruence with the tribal culture and empower these marginalized. We analysed data from Health Management Information System from the government of India for the year 2013-2014 and compared to assess the functionality. Findings Our study revealed that many public healthcare facilities deviated from the recommended safe medical practices. The district hospital (Balasore) and the community health centre (Hatigarh), which provide basic and referral services in Jaleswar block, do not comply with and fall short of the minimum requirement prescribed by the WHO guidelines. It is felt that the 
state is attempting to implement maternal health programme uniformly across its territory without considering the cultural specificity of the tribal pockets. This is well visible in the capacity-building curriculum where specific strategies to work in hard-to-reach areas are not addressed. Subsequently, ASHAs fail to reach the pregnant women effectively, disseminate good practices and motivate them for adequate health care. Another illustration of the tailor-made strategy of the state is the way Health Management Information System is designed.

Discussion In order to comply with national standards, the state has failed to incorporate traditional practices that largely fall outside the recommended scientific procedure. Despite their cultural confirmation and deep indigenous value, they are dismissed as trivial practices. Resultantly, health plans that are based on information generated through Health Management Information System do not incorporate cultural variations. Strategies that follow from health plans are mainly target-driven rather than reinforcing indigenous practices.

No competing interest. 